\title{
z Sonstem \\ LESS IS MORE? - A PILOT RCT OF FLUID STRATEGY IN CRITICALLY ILL CHILDREN WITH ACUTE RESPIRATORY TRACT INFECTION
}

\section{Sarah A. Ingelse}

Department of Pediatric Intensive Care, Emma Children's Hospital, Amsterdam UMC, University of Amsterdam, Amsterdam, The Netherlands https://orcid.org/0000-0002-6516-9830

\section{Vincent G. Geukers}

Department of Pediatric Intensive Care, Emma Children's Hospital, Amsterdam UMC, University of Amsterdam, Amsterdam, The Netherlands

\section{Monique E. Dijsselhof}

Department of Dietetics, Emma Children's Hospital, Amsterdam UMC, Amsterdam, The Netherlands Joris Lemson

Department of Intensive Care, Radboud University Medical Center, Nijmegen, The Netherlands

\section{Reinout A. Bem}

Department of Pediatric Intensive Care, Emma Children's Hospital, Amsterdam UMC, University of Amsterdam, Amsterdam, The Netherlands

\section{Job B. van Woensel}

Department of Pediatric Intensive Care, Emma Children's Hospital, Amsterdam UMC, University of Amsterdam, Amsterdam, The Netherlands

\section{Research Article}

\section{Keywords:}

Posted Date: November 30th, 2018

DOI: https://doi.org/10.21203/rs.2.31/v1

License: (c) (i) This work is licensed under a Creative Commons Attribution 4.0 International License. Read Full License

Version of Record: A version of this preprint was published at Frontiers in Pediatrics on December 10th, 2019. See the published version at https://doi.org/10.3389/fped.2019.00496. 


\section{Abstract}

Background: Fluid overload is common in critically ill children and is associated with adverse outcome. Therefore, restricting fluid intake may be beneficial. This study aims to pilot the feasibility of a RCT comparing a conservative to a standard, more liberal, strategy of fluid management in mechanically ventilated (MV) pediatric patients with acute respiratory tract infection (ARTI). Methods: This is a pilot RCT and feasibility study in a single, tertiary referral pediatic intensive care unit. Twenty-three children receiving MV for ARTI, without ongoing hemodynamic support, admitted to the PICU of the Emma Children's Hospital/Amsterdam UMC between 2016-2018 were included. Patients were randomized to a conservative ( $<70 \%$ of normal intake) or standard ( $>85 \%$ of normal intake) fluid strategy, which was kept throughout the period of MV. Results: Primary endpoints were adherence to fluid strategy and safety parameters such as calorie and protein intake. Secondary outcomes were cumulative fluid intake (CFI) and cumulative fluid balance (CFB) on day 3 . In the conservative group, in $75 \%$ of the MV days patients reached their target fluid intake. Median calorie intake over all MV days was 67.8 [53.1-75.2] kcal/kg/day and 1.6 [1.3-1.7] gr protein/ $\mathrm{kg} /$ day (no difference between groups). No adverse effects on hemodynamics or electrolyte imbalances were noted. Mean CFI on day 3 was $262.3( \pm 58.9) \mathrm{ml} / \mathrm{kg}$ in the conservative group vs. $360.5( \pm 52.6) \mathrm{ml} / \mathrm{kg}$ in the standard fluid group ( $p<0.001)$, which did not result in a lower CFB. Conclusions: A conservative fluid strategy in MV children with ARTI seems feasible, without limiting metabolic needs. However, in our study a conservative fluid strategy surprisingly did not reduce the degree of fluid overload. This pilot study aids the design and sample size calculation of a future larger multicenter RCT, in which we need to redefine the target fluid strategy, possibly by even further fluid restriction and early initiation of active diuresis. Trial registration: ClinicalTrials.gov, NCT02989051. Registered 12 December 2016 - Retrospectively registered, https://www.clinicaltrials.gov/ct2/show/NCT02989051

\section{Background}

Fluid overload is a major problem in critically ill patients and is gaining increasing attention in both research and clinical practice. Importantly, body fluid mostly accumulates early in the disease process (1, 2), which is believed to occur due to multiple factors, such as overzealous intravenous fluid loading, proinflammatory injury with systemic capillary leak and cardiopulmonary dysfunction during critical illness. Fluid overload results in interstitial fluid retention, including formation of pulmonary edema, compromising alveolar-capillary oxygen diffusion. Numerous studies have shown that - in both adults and children - fluid overload, or extreme positive cumulative fluid balance, has adverse effects on outcome, such as a longer duration of mechanical ventilation and even higher mortality rates (2-6). Of great interest, a large RCT in adult ARDS patients has shown a conservative fluid management regimen to lead to more ventilator-free days $(7,8)$. Whether this also holds true for pediatric patients remains yet unknown.

As evidence in the pediatric population up to this point is exclusively mounted through observational studies, it becomes clear that there is a great need for prospective testing in children $(4,9)$. However, 
studies in children come with considerable difficulties such as small patient populations and ethical issues. Moreover, critically ill children are prone to cumulative energy and protein deficits during PICU admission, due to both insufficient amounts of prescribed calories and unreliable predictive requirement equations $(10,11)$, with $50 \%$ of cumulative protein energy malnutrition developing in the first 48 hours of admission (10). Negative protein balance with loss of lean body mass is associated with a higher risk of infections, persisting critical illness, and increased length of stay in the $\operatorname{PICU}(12,13)$. Restrictive fluid management may add to this risk. In order to reach positive protein balance, the minimum nutritional requirements for critically ill children are found to be $57 \mathrm{kCal} / \mathrm{kg} /$ day and $1.5 \mathrm{~g} / \mathrm{kg} /$ day protein intake (14). Along with the logistical difficulty of reaching adequate study sample sizes, previous suggestions of the use of Bayesian statistical approaches (4) might aid to enhance the power and lower the necessary sample size for these studies. These issues together raise the need for pilot studies in critically ill children.

In this study we performed a pilot RCT to determine the feasibility and safety of a larger multicenter RCT comparing current fluid maintenance protocols with an early conservative fluid management strategy. Primary endpoints were cumulative fluid intake (CFI), cumulative fluid balance (CFB) on day 3 and adherence to fluid strategy. We chose a highly prevalent patient cohort of mechanically ventilated critically ill children with ARTI, not necessarily characterized by major hemodynamic instability and capillary leak, in order to steer towards a general PICU fluid management protocol.

\section{Methods}

This is a single center feasibility and pilot RCT in patients admitted to the PICU of the Emma Children's Hospital/Amsterdam UMC, The Netherlands. Our PICU is a 12-bed, tertiary unit, serving the greater Amsterdam area in The Netherlands. Patients were enrolled between September 2016 and April 2018.

They were randomized in a 1:1 allocation ratio to a conservative fluid regimen or a standard, more liberal, fluid regimen in randomly permuted blocks with the use of an encrypted and automated website (Sealed Envelope $\left.^{\mathrm{TM}}\right)$. This study was approved by the Medical Ethical Review board of the Amsterdam UMC. Written informed consent was obtained from parents or guardians of eligible patients. A Safety Monitoring board was set up to monitor monthly progress and possible relation with adverse events in both study arms.

\section{Inclusion criteria}

Patients below 10 years of age were eligible when they were intubated and mechanically ventilated for a (suspected) acute lower respiratory tract infection, with an anticipated duration of mechanical ventilation of at least 72 hours at enrolment. Enrolment into the study protocol needed to be fulfilled within 12 hours after start of mechanical ventilation.

\section{Exclusion criteria}


Exclusion criteria were use of previous or maintenance diuretic treatment at enrolment, ongoing (fluid) resuscitation at enrolment, acute kidney injury with the need for renal replacement therapy and patients in need of a particular fluid regimen (i.e. for their medical history such as cardiovascular disease and/or congenital heart disease).

\section{Study protocol}

In this simple study protocol, patients received either a conservative fluid regimen which consisted of (less than) $70 \%$ of normal fluid recommendations in a healthy child, or a standard, more liberal, fluid regimen of (more than) $85 \%$ of normal recommendations (Table 1) $(15,16)$. The percentual cut-off for the standard fluid strategy was chosen based on clinical practice at the time of study initiation. Fluid treatment regimens were set to guarantee the caloric and protein requirements as described by Bechard et al (14).

\section{Table 1: Fluid strategies per randomization arm per kg bodyweight.}




\begin{tabular}{|c|c|c|c|c|c|}
\hline $\begin{array}{l}\text { Weight } \\
\text { (kg) }\end{array}$ & $\begin{array}{l}\text { Normal fluid } \\
\text { recommendations } \\
\text { (ml/kg/day) }\end{array}$ & $\begin{array}{l}\text { Conservative: } \\
70 \% \text { fluid } \\
\text { restriction } \\
\text { (ml/kg/day) }\end{array}$ & $\begin{array}{l}\text { Standard: }>85 \% \text { of } \\
\text { normal } \\
\text { recommendations } \\
\text { (ml/kg/day) }\end{array}$ & $\begin{array}{l}\text { Guarantee of } \\
\text { kcal intake } \\
\text { (kcal/kg/day) }\end{array}$ & $\begin{array}{l}\text { Guarantee } \\
\text { of protein } \\
\text { intake } \\
\text { (g/kg/day) }\end{array}$ \\
\hline $0-6$ & 150 & 105 & $>128$ & 75 & 1.5 \\
\hline $6-7.5$ & 130 & 91 & $>111$ & 75 & 1.5 \\
\hline $7.5-9$ & 120 & 84 & $>102$ & 75 & 1.5 \\
\hline $9-10$ & 110 & 77 & $>94$ & 75 & 1.5 \\
\hline 11 & 95 & 67 & $>81$ & 60 & 1.5 \\
\hline 12 & 92 & 64 & $>78$ & 60 & 1.5 \\
\hline 13 & 88 & 62 & $>75$ & 60 & 1.5 \\
\hline 14 & 86 & 60 & $>73$ & 60 & 1.5 \\
\hline 15 & 83 & 58 & $>71$ & 60 & 1.5 \\
\hline 16 & 81 & 57 & $>69$ & 60 & 1.5 \\
\hline 17 & 79 & 56 & $>67$ & 60 & 1.5 \\
\hline 18 & 78 & 54 & $>66$ & 60 & 1.5 \\
\hline 19 & 76 & 53 & $>65$ & 60 & 1.5 \\
\hline 20 & 75 & 53 & $>64$ & 60 & 1.5 \\
\hline 21 & 72 & 51 & $>61$ & 60 & 1.5 \\
\hline 22 & 70 & 49 & $>60$ & 60 & 1.5 \\
\hline 23 & 68 & 47 & $>58$ & 60 & 1.5 \\
\hline 24 & 66 & 46 & $>56$ & 60 & 1.5 \\
\hline 25 & 64 & 45 & $>54$ & 60 & 1.5 \\
\hline 26 & 62 & 44 & $>53$ & 60 & 1.5 \\
\hline 27 & 61 & 43 & $>52$ & 60 & 1.5 \\
\hline 28 & 59 & 42 & $>50$ & 60 & 1.5 \\
\hline 29 & 58 & 41 & $>49$ & 60 & 1.5 \\
\hline 30 & 57 & 40 & $>48$ & 60 & 1.5 \\
\hline$>30$ & 50 & 35 & $>43$ & 60 & 1.5 \\
\hline
\end{tabular}

Normal fluid recommendations of infants and young children are based on Shaw (15). 
During the study period, the attending physician was free to start diuretic treatment when clinically needed, or even make adaptations in the allocated fluid treatment when there were valid clinical reasons to do so.

\section{Data collection}

Data were prospectively collected from the patient clinical data management system. Primary outcomes were cumulative fluid intake (CFI) and feasibility parameters such as adherence to the fluid intake corresponding to the allocated fluid strategy. Adherence was observed for every study day for each patient and median adherence over all study days was calculated in a percentage of the total amount of study days. Reasons for non-adherence were examined for the conservative fluid strategy as this is the most challenging intervention with respect to limiting fluid volume for medication and nutrition. Fluid intake, fluid output and fluid balance were collected daily for the whole period of mechanical ventilation. Fluid intake included intravenously administered fluids and medication, parenteral and enteral feeding, oral medication and blood products. Fluid output included urine output, stools, gastric aspirate, blood loss, fluid loss from drains and losses from other body cavities. Fluid balance was calculated over every 24 hours as intake minus output per kilogram $(\mathrm{kg})$ body weight on admission. Secondary feasibility parameters were calorie and protein intake, the need for interventions such as fluid boluses or administration of diuretics. Calorie and protein intake were assessed daily with the help of an experienced dietician. To assess hemodynamic stability in either fluid strategy, blood pressure and heart rate were monitored continuously. In order to evaluate electrolyte imbalances, available sodium, potassium and chloride assays were checked for too low or high values. An electrolyte imbalance was scored if the levels of the respective electrolytes were too high or low for more than 1 day consecutively during admission (normal ranges used: sodium 135-145 mmol/L; potassium 3.5-5.2 mmol/L; chloride 96-111 mmol/L). To assess the occurrence of acute kidney injury (AKI) the KDIGO AKI criteria were used (17) by using the urine output criteria. Serum creatinine was not used as this was not readily available at baseline in many children. In the patient data management system, ventilation and oxygenation variables were recorded continuously and validated at least hourly. The Pediatric Index of Mortality (PIM) 2 score was calculated from each patient to assess for severity of illness. To assess the severity of oxygenation defect in our patients, the oxygen saturation index (OSI) was calculated each morning as OSI $=([\mathrm{Paw} \times \mathrm{FiO} 2] / \mathrm{SpO} 2) \mathrm{x}$ 100 , only when SpO2 was below $97 \%$ (18).

\section{Statistical analysis}

All analyses were performed according to the intention-to-treat principle. A two-sided p-value of 0.05 was considered as statistically significant. Statistical analyses were performed using R statistical programming software (19) and the 'Ime4' (20) package was used for mixed-effects model analyses. A linear mixed-effects model with a random intercept and slope per subject was used to analyse the effect of time (as a continuous variable) and fluid strategy on blood pressure and heart rate measured at hourly intervals. Patient characteristics are described by descriptive statistics. Student's t-test or Mann-Whitney U 
tests are used to compare continuous variables. Fisher's exact test was used for categorical variables and proportions. Data are expressed as means $( \pm S D)$, medians [IQR] or proportions as appropriate.

\section{Results}

\section{Patient characteristics}

During the study period 37 patients were screened for enrollment. 24 patients gave their consent for inclusion, but one patient did not start the study protocol due to referral to another hospital. Therefore, in total 23 patients were included and enrolled in the study protocol. Of these, 12 were randomized to the conservative fluid arm and 11 in the standard fluid arm. Table 2 shows their baseline and clinical characteristics.

Table 2: Baseline demographic and clinical characteristics of mechanically ventilated patients with acute respiratory tract infection.

\begin{tabular}{|c|c|c|c|}
\hline \multirow[t]{4}{*}{ Variable } & \multirow{4}{*}{$\begin{array}{l}\text { Overall } \\
(\mathrm{N}=23)\end{array}$} & \multirow{3}{*}{$\begin{array}{l}\text { Fluid strategies } \\
\begin{array}{l}\text { Conservative } \\
\text { group }\end{array}\end{array}$} & \multirow[b]{3}{*}{$\begin{array}{l}\text { Standard } \\
\text { group }\end{array}$} \\
\hline & & & \\
\hline & & & \\
\hline & & $(N=12)$ & $(N=11)$ \\
\hline Age (months), median [IQR] & $\begin{array}{l}2.7[0.8- \\
10.1]\end{array}$ & $3.4[0.8-11.5]$ & $2.7[0.8-6.7]$ \\
\hline Sex, male, N (\%) & $13(57)$ & $6(50)$ & $7(64)$ \\
\hline Admission weight in kg, median [IQR] & $\begin{array}{l}5.5[3.9- \\
8.2]\end{array}$ & $5.7[4.3-9.8]$ & $5.5[3.4-8.2]$ \\
\hline History of chronic illnessa, N (\%) & $4(17.4)$ & $4(33.3)$ & $0(0)$ \\
\hline RSV positive, N (\%) & $17(73.9)$ & $11(91.7)$ & $6(54.5)$ \\
\hline Bacterial (super)infectionb, N (\%) & $9(39.1)$ & $6(50.0)$ & $3(27.3$ \\
\hline Pediatric Index of Mortality 2 score, median [IQR] & $\begin{array}{l}1.3[1.1- \\
2.1]\end{array}$ & $1.3[1.0-2.2]$ & $1.5[1.1-2.1]$ \\
\hline $\begin{array}{l}\text { Oxygen saturation index at day of admission, } \\
\text { median [IQR] }\end{array}$ & $\begin{array}{l}6.1[4.0- \\
7.4]\end{array}$ & $6.6[3.9-8.1]$ & $6.1[4.1-6.5]$ \\
\hline
\end{tabular}

a Chronic illness included prematurity with bronchopulmonary dysplasia, muscle-eye-brain disease and epilepsy. 
b Positive cultures of tracheal aspirate or bronchial lavage fluid included one or more of the following bacteria: Haemophilus influenzae, Moraxella catarrhalis, Streptococcus pneumoniae, Staphylococcus aureus.

Median age was 2.7 months [IQR 0.8-10.1] and was distributed evenly among the treatment groups. There was no difference in the distribution of sex between fluid strategies. Patients had an average median [IQR] admission weight of $5.5 \mathrm{~kg}$ [3.9-8.2], which was also similar in both fluid strategy groups. There were no other baseline differences of parameters indicating severity of disease, such as the PIM2 score or OSI, between the fluid strategy groups (Table 2).

\section{Feasibility}

In this pilot study, an enrollment rate of $62 \%$ was reached (23/37 patients screened for participation). The main reasons for non-inclusion were because parents declined participation. Three patients were missed, and two patients were not asked for permission because the attending physician considered the emotional burden of their child's disease already too demanding to ask for consent. During $75 \%$ of the study days, mechanically ventilated children in the conservative group received fluid according to their target intake. There was no significant difference in adherence between both fluid management groups (Table 3).

Table 3: Feasibility and safety parameters per fluid strategy.

\begin{tabular}{|c|c|c|c|}
\hline Parameter & $\begin{array}{l}\text { Conservative group } \\
(n=12)\end{array}$ & $\begin{array}{l}\text { Standard group } \\
(n=11)\end{array}$ & $\begin{array}{l}\text { P- } \\
\text { value }\end{array}$ \\
\hline $\begin{array}{l}\text { Adherence to fluid intake, \% of study days; } \\
\text { median [IQR] }\end{array}$ & $75.0[50.0-96.4]$ & $66.7[40.0-100.0]$ & 0.474 \\
\hline Calorie intake, kcal/kg; median [IQR] & $67.9[51.5-74.0]$ & $67.2[58.0-75.2]$ & 0.878 \\
\hline Protein intake, gr protein/kg; median [IQR] & $1.6[1.3-1.8]$ & $1.5[1.2-1.7]$ & 0.598 \\
\hline $\begin{array}{l}\text { Cumulative furosemide day } 3, \mathrm{mg} / \mathrm{kg} \text {; } \\
\text { median [IQR] }\end{array}$ & $0.9[0.08-2.2]$ & $0.6[0.0-1.0]$ & 0.361 \\
\hline $\begin{array}{l}\text { Cumulative diuresis at day } 3 \text {, in } \mathrm{ml} / \mathrm{kg} \text {; mean } \\
\pm \mathrm{sd}\end{array}$ & $174.1( \pm 55.5)$ & $265.0( \pm 36.6)$ & $<0.001$ \\
\hline Patients who received fluid boluses, N (\%) & $3(25.0)$ & $3(27.3)$ & 1.000 \\
\hline Acute Kidney Injury (AKI), grade I, N (\%) & $4(33.3)$ & $0(0)$ & 0.093 \\
\hline
\end{tabular}

No significant differences in the feasibility and safety parameters occurred between the two groups. Diuresis was significantly higher in the standard group. IQR = interquartile range.

Figure 1 portrays adherence in percentage of the normal fluid intake recommendations, which were also used for developing the fluid arms (Table 1). Patients in the conservative arm needed to stay below $70 \%$ of this recommendation, while patients in the standard fluid strategy were deemed to receive fluids above 
$85 \%$ of this recommendation. Of the patients in the conservative fluid strategy group who ran over their target intake, $77.8 \%$ was due to too high parenteral intake either because of high medication needs (43\%), receiving extra glucose parenterally when enteral feeding was not yet well tolerated $(29 \%)$ or occasionally extra fluid boluses $(21 \%)$ were given.

Calorie and protein intake were not significantly different between fluid strategy groups and reached an overall median of 67.8 [53.1-75.2] kcal/ $/ \mathrm{kg}$ and 1.6 [1.3-1.7] gr protein $/ \mathrm{kg}$ for all patients. The need for interventions such as administration of diuretics or fluid boluses was similar in both fluid groups. Yet cumulative diuresis was significantly higher in the standard fluid group $(p<0.001)$. Importantly, a conservative fluid strategy did not lead to worsened hemodynamic response, as measured by mean blood pressure, during the mechanical ventilation period $(p=0.687)$. There were no significant differences in electrolyte imbalances between the fluid strategies (Table 4).

\section{Table 4: Electrolyte imbalances}

\begin{tabular}{llll} 
Parameter & Conservative group $(\mathbf{n = 1 2 )}$ & Standard group $(\mathbf{n = 1 1 )}$ & P-value \\
\hline Hyponatremia, N (\%) & $5(41.7)$ & $3(27.3)$ & 0.667 \\
\hline Hypernatremia, N (\%) & $1(8.3)$ & $0(0)$ & 1.000 \\
\hline Hypokalemia, N (\%) & $2(16.7)$ & $0(0)$ & 0.478 \\
\hline Hyperkalemia, N (\%) & $3(25.0)$ & $4(36.4)$ & 0.667 \\
\hline Hypochloremia, N (\%) & $4(33.3)$ & $1(9.1)$ & 0.317 \\
\hline Hyperchloremia, N (\%) & $0(0)$ & $0(0)$ & -
\end{tabular}

No significant differences in the occurrence of imbalances were found between the two groups. Electrolytes were deemed out of balance when values were out of the normal range for more than one day consecutively. Normal ranges used were: sodium 135-145 mmol/L; potassium 3.5-5.2 mmol/L; chloride $96-111 \mathrm{mmol} / \mathrm{L}$.

\section{Fluid intake}

Despite a significant difference in fluid intake between the allocated fluid strategies, no significant difference in cumulative fluid balance on day 3 was found $(79.7 \pm 19.7 \mathrm{vs}$. $84.2 \pm 33.0 \mathrm{ml} / \mathrm{kg}$; $\mathrm{p}=0.682$, Figure 2). Body weight on day 3 of mechanical ventilation was available for 11 children ( $48 \%$ of all study patients) and for these patients the change in body weight over the first 3 days of admission could be calculated. Measuring body weight is considered quite burdensome for mechanically ventilated critically ill patients, which led to the low percentage of available weights at day 3 . In the conservative group, the median weight change was $+3.1 \%[-0.6-8.1]$ versus $+1.8 \%[-1.6-10.3]$ in the standard group $(p=1.000)$. Cumulative parenteral fluid intake on day 3 consisted of $88.8( \pm 29.6) \mathrm{ml} / \mathrm{kg}$ versus $115.6( \pm 39.7) \mathrm{ml} / \mathrm{kg}$ in the conservative and standard fluid strategy respectively $(p=0.079)$, translating into a percentage of 
$33.9 \%$ and $32,1 \%$ of total CFI on day 3 in either group. Urine output was significantly higher in the standard group ( $p<0.001$, Figure 2 ; Table 3 ). In the conservative group, 4 out of 12 patients $(33.3 \%$ ) had a diuresis of less than $0.5 \mathrm{ml} / \mathrm{kg} /$ hour for 8 hours consecutively, defining AKI stage I (17), opposed to zero patients in the standard group $(p=0.093$; Table 3$)$.

\section{Clinical outcome}

The duration of mechanical ventilation was 5.1 [4.7-7.0] days in the conservative group and 4.3 [2.7-5.4] days in the standard fluid group $(p=0.044)$. There was no significant difference between fluid strategies in oxygenation failure over time, as measured by OSI $(p=0.6172)$.

\section{Discussion}

In this single center pilot RCT we investigated the feasibility of conducting a large-scale trial comparing the current standard, more liberal, fluid maintenance strategy to a conservative fluid strategy during mechanical ventilation in critically ill pediatric patients with ARTI. Employing a simple protocol based on real-life practice, without extensive logarithms or flowcharts, we were able to significantly reduce fluid intake in these critically ill mechanically ventilated children, wherein moderate adherence was reached, without impairment of calorie and protein intake. In current literature, the association of fluid overload with clinical outcome in critically ill children has only been estimated through retrospective cohort studies $(2,4,21,22)$. This study provides a first instigation in research towards determining a possible causative relationship between fluid overload and worse outcome in the PICU.

Different fluid strategies led to a large difference in fluid intake, yet surprisingly no difference in cumulative fluid balance or weight gain was reached. Although patients in the standard arm did not receive higher dosages of diuretics, they did have a significantly higher cumulative diuresis. This indicates that patients were still capable of losing excess fluids without the aid of additional medication. What's more, it suggests that patients in the standard arm received more fluid than was necessary for their clinical situation. In this cohort more than $70 \%$ of the patients were infants with respiratory failure due to viral-induced ARTI, which is in general a population without multiple organ failure (including severe kidney injury) and/or major capillary leak. Apparently, these patients are still able to maintain adequate diuresis and fluid homeostasis. Yet, in an earlier study we found that this specific patient group also suffers from fluid overload which is associated with prolonged duration of mechanical ventilation (6). Nevertheless, this pilot study was not powered to assess the effect of fluid strategy on clinical outcome yet and further research is required to draw conclusions on this matter.

Zooming in on feasibility, we found that a vast majority of patients reached their targeted fluid intake. When fluid intake exceeded the targeted intake, this was mostly due to too high parenteral load if patients did not yet tolerate the necessary enteral feeding or if they had high medication needs. Although care was taken to minimize volume and concentrate all given solutions, it remains possible that errors were made and further restriction is possible in the future. Important pillars in determining feasibility are numerous 
safety parameters, such as energy intake. It seems that feeding was adequately concentrated in the conservative patients, resulting in a similar calorie and protein intake in both groups.

Taken together, these outcomes imply to us that there might be room for further fluid restriction, possibly by combining low fluid volume administration with early start of active diuretic treatment. A recent implementation study in children with ARDS or sepsis has shown that the combination of fluid restriction, drug volume reduction, dynamic monitoring of preload markers to determine the need for fluid bolus administration, early use of diuretics and early initiation of enteral feeds decreased fluid overload and MV duration (23). Yet, this study compared the post-implementation outcomes to a retrospective cohort and its results will therefore be inferior to a (large multicenter) RCT. In our study we found a small significant difference in MV duration favoring the standard fluid treatment over conservative fluids. However, we must stress that this was in essence a pilot for feasibility, which was not powered to assess the effect on clinical outcome.

In order to set up a multicenter RCT in the future, we need to take a close look at the needed sample size to obtain sufficient power to rule out any uncertainties in this pediatric population. Previously, Bayesian statistical modelling has been proposed to set up further pediatric studies to minimize the number of children needed to be enrolled and still reach adequate power (4). Bayesian statistical modelling has already been used in different ways in the pediatric field (24). Based on the article by Schoenfeld et al. (25), power and sample size can be calculated using data from adult trials. We used the FACTT trial's (7) main outcome effect, i.e. ventilator-free days, to calculate the necessary sample size. Estimating a similar true effect in children of a conservative fluid strategy, with a standard deviation of 1 to describe the variation between the effect of pediatric and adult effects, we calculated that 250 pediatric patients are necessary to reach a power of $>80 \%$. Conversely, without the addition of the adult data through Bayesian modelling, only a power of $37 \%$ would be achieved for this $N$. Without a Bayesian approach, approximately 800 patients would be necessary to achieve the same power.

Now that we know some pilot data from this study, the next question is how to set up the different fluid strategies for a future multicenter RCT. This pilot proves that a conservative fluid management strategy is feasible and safe both hemodynamically and with regard to nutritional intake. Moreover, the standard, liberal fluid strategy led to higher diuresis but similar fluid balances, making it a potential harmful form of overtreating our patients. We propose a conservative fluid regimen which consists of compensation for insensible losses, which are significantly lower in mechanically ventilated patients (26), and a surplus of volume for medication, feeding and securing kidney function. Securing kidney function should be treated with caution to prevent (severe) AKI from occurring. In addition to fluid volume restriction, early start of active diuretic treatment should be considered. Altogether, this creates a tailor-made and patient-targeted fluid management strategy.

In conclusion, a conservative fluid strategy in mechanically ventilated children with ARTI seems feasible, without limiting metabolic needs. We deem it justified to set up a larger multicenter RCT in this specific patient population, be it with an adapted protocol of further fluid restriction and early start of active 
diuresis treatment. Bayesian statistical modelling may be a solution to provide us with adequate sample size and power for this future RCT.

\section{Declarations}

\section{Ethics approval and consent to participate}

This study was approved by the Medical Ethical Review board of the Amsterdam UMC [NL55053.018.16]. Written informed consent for participation was obtained from parents or guardians of eligible patients.

\section{Consent for publication}

Consent for publication was obtained upon written informed consent from parents or guardians of eligible patients.

\section{Availability of data}

The datasets used and/or analysed during the current study are available from the corresponding author on reasonable request.

\section{Competing interests}

The authors declare that they have no competing interests.

\section{Funding}

This research was supported by the Ammodo Foundation and the Foundation of Pediatric Intensive Care (SKIC) in the Netherlands. The sponsor had no role in the design and execution of the research, the writing of the report or the decision to submit the manuscript for publication.

\section{Authors' contributions}

SI designed the study, included and randomized patients and optimized their fluid intake accordingly. SI also analyzed and interpreted the data and wrote the manuscript. VG helped design the study specifically focusing on the calorie and protein intake requirements. Moreover, he contributed in writing the manuscript. MD focused specifically on designing the calorie and protein intake requirements and provided advice during the study on how to optimize energy intake during study participation. JL interpreted the data and provided major contribution in writing the manuscript. RB designed the study, interpreted the data and was a major contributor in writing the manuscript. JvW designed the study, interpreted the data and also provided major contribution in writing the manuscript. All authors read and approved the final manuscript.

\section{Acknowledgements}


This research was supported by the Ammodo Foundation and the Foundation of Pediatric Intensive Care (SKIC) in the Netherlands.

\section{References}

1. Rosenberg AL, Dechert RE, Park PK, et al. Review of a large clinical series: association of cumulative fluid balance on outcome in acute lung injury: a retrospective review of the ARDSnet tidal volume study cohort. J Intensive Care Med. 2009;24(1):35-46.

2. Valentine SL, Sapru A, Higgerson RA, et al. Fluid balance in critically ill children with acute lung injury. Criti Care Med. 2012;40(10):2883-9.

3. Alobaidi R, Morgan C, Basu RK, et al. Association Between Fluid Balance and Outcomes in Critically III Children: A Systematic Review and Meta-analysis. JAMA Pediatr. 2018;172(3):257-68.

4. Flori HR, Church G, Liu KD, et al. Positive fluid balance is associated with higher mortality and prolonged mechanical ventilation in pediatric patients with acute lung injury. Crit Care Res Pract. 2011;2011:854142.

5. Sakr Y, Vincent JL, Reinhart K, et al. High tidal volume and positive fluid balance are associated with worse outcome in acute lung injury. Chest. 2005;128(5):3098-108.

6. Ingelse SA, Wiegers HM, Calis JC, et al. Early Fluid Overload Prolongs Mechanical Ventilation in Children With Viral-Lower Respiratory Tract Disease. Pediatr Crit Care Med. 2017;18(3):e106-e11.

7. Wiedemann HP, Wheeler AP, Bernard GR, et al. Comparison of two fluid-management strategies in acute lung injury. N Engl J Med. 2006;354(24):2564-75.

8. Grissom CK, Hirshberg EL, Dickerson JB, et al. Fluid management with a simplified conservative protocol for the acute respiratory distress syndrome*. Crit Care Med 2015;43(2):288-95.

9. Flori HR. Tipping the Scales With Fluid Overload in Pediatric Bronchiolitis: When Will We and What Can We Learn From Here? Pediatr Crit Care Med. 2017;18(3):289-90.

10. Hulst JM, van Goudoever JB, Zimmermann LJ, et al. The effect of cumulative energy and protein deficiency on anthropometric parameters in a pediatric ICU population. Clin Nutr. 2004;23(6):1381-9.

11. Oosterveld MJ, Van Der Kuip M, De Meer K, et al. Energy expenditure and balance following pediatric intensive care unit admission: a longitudinal study of critically ill children. Pediatr Crit Care Med. 2006;7(2):147-53.

12. Cogo PE, Carnielli VP, Rosso F, et al. Protein turnover, lipolysis, and endogenous hormonal secretion in critically ill children. Crit Care Med. 2002;30(1):65-70. 
13. Leite HP, Isatugo MK, Sawaki L, et al. Anthropometric nutritional assessment of critically ill hospitalized children. Rev Paul Med. 1993;111(1):309-13.

14. Bechard LJ, Parrott JS, Mehta NM. Systematic review of the influence of energy and protein intake on protein balance in critically ill children. J Pediatr. 2012;161(2):333-9.e1.

15. Shaw V. Clinical Paediatric Dietetics. 4th edition. ed. Oxford: Wiley Blackwell Science; 2015.

16. Scientific Advisory Committee on Nutrition. Dietary Reference Values for Energy 2011 [updated 1 November 2011. Independent report]. Available from: https://www.gov.uk/government/publications/sacndietary-reference-values-for-energy.

17. Selewski DT, Cornell TT, Heung M, et al. Validation of the KDIGO acute kidney injury criteria in a pediatric critical care population. Intensive Care Med. 2014;40(10):1481-8.

18. Khemani RG, Rubin S, Belani S, et al. Pulse oximetry vs. PaO2 metrics in mechanically ventilated children: Berlin definition of ARDS and mortality risk. Intensive Care Med. 2015;41(1):94-102.

19. R Core Team. R: A language and environment for statistical computing. . R Foundation for Statistical Computing, Vienna, Austria. 2017.

20. Bates DMM, Bolker B, Walker S. Fitting Linear Mixed-Effects Models Using Ime4. J Stat Soft. 2015(67(1)):1-48.

21. Garcia PCR, Korb C, Piva JP, et al. The effects of positive fluid balance in infants younger than one year with bronchiolitis undergoing mechanical ventilation. Pediatr Crit Care Med. 2014;1):97.

22. Sinitsky L, Walls D, Nadel S, et al. Fluid Overload at 48 Hours Is Associated With Respiratory Morbidity but Not Mortality in a General PICU: Retrospective Cohort Study*. Pediatr Crit Care Med. 2015;16(3):205-9.

23. Diaz F, Nunez MJ, Pino P, et al. Implementation of preemptive fluid strategy as a bundle to prevent fluid overload in children with acute respiratory distress syndrome and sepsis. BMC Pediatr. 2018;18(1):207.

24. Modesto IAV, Khemani RG, Medina A, et al. Bayes to the Rescue: Continuous Positive Airway Pressure Has Less Mortality Than High-Flow Oxygen. Pediatr Crit Care Med. 2017;18(2):e92-e9.

25. Schoenfeld DA, Hui Z, Finkelstein DM. Bayesian design using adult data to augment pediatric trials. Clin Trials. 2009;6(4):297-304.

26. Raman S, Peters M. Fluid management in the critically ill child. Pediatr Nephrol. 2014;29(1):23-34.

\section{Figures}




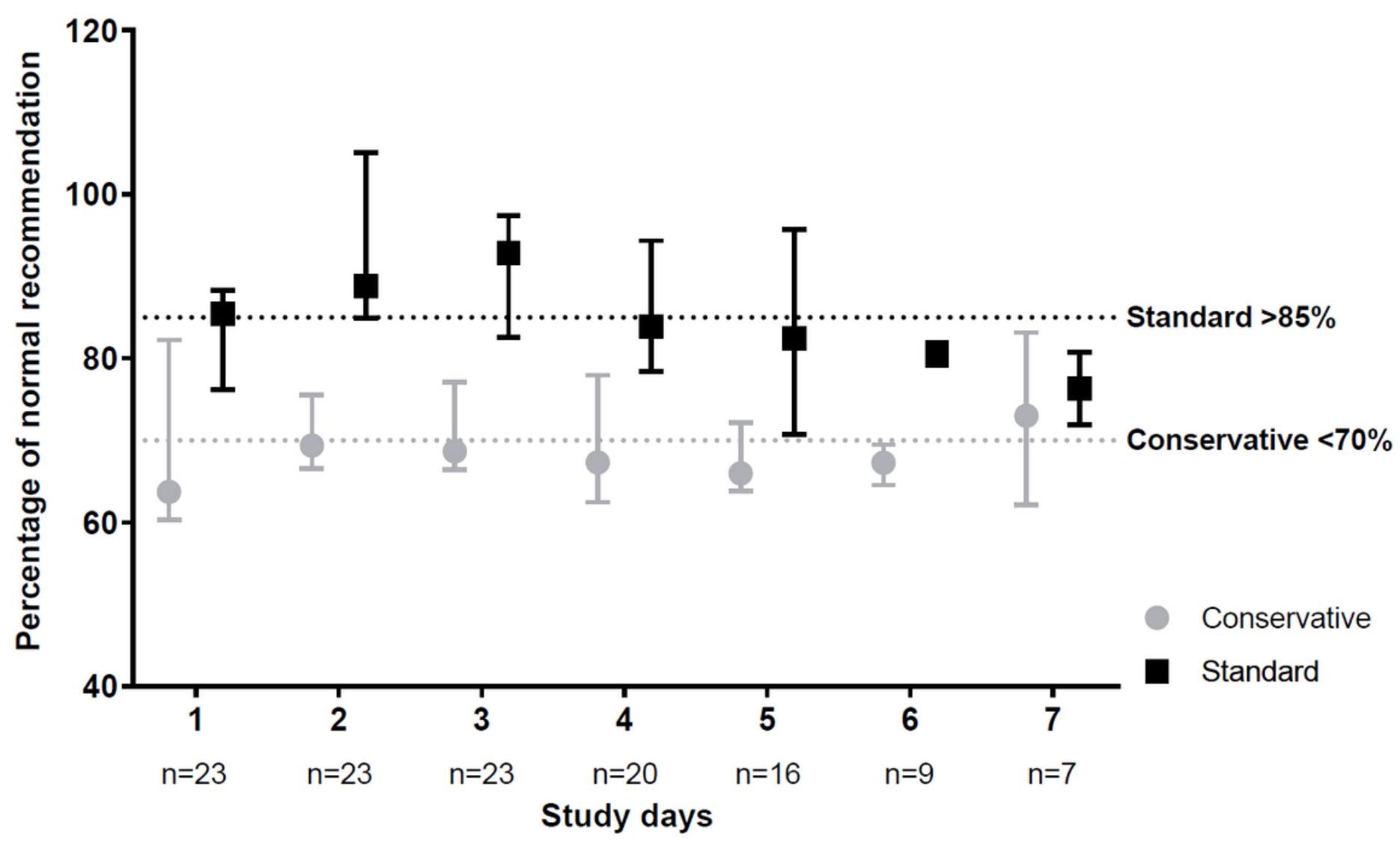

Figure 1

Fluid intake per fluid strategy in percentage of normal fluid recommendation. Normal fluid recommendations are based on Shaw (15). Patients in the standard fluid strategy were allocated to stay above $85 \%$ of this fluid volume. Patients in the conservative fluid strategy were deemed to stay below $70 \%$ of this recommendation. Plot depicts median with interquartile ranges over all patients in each group. 


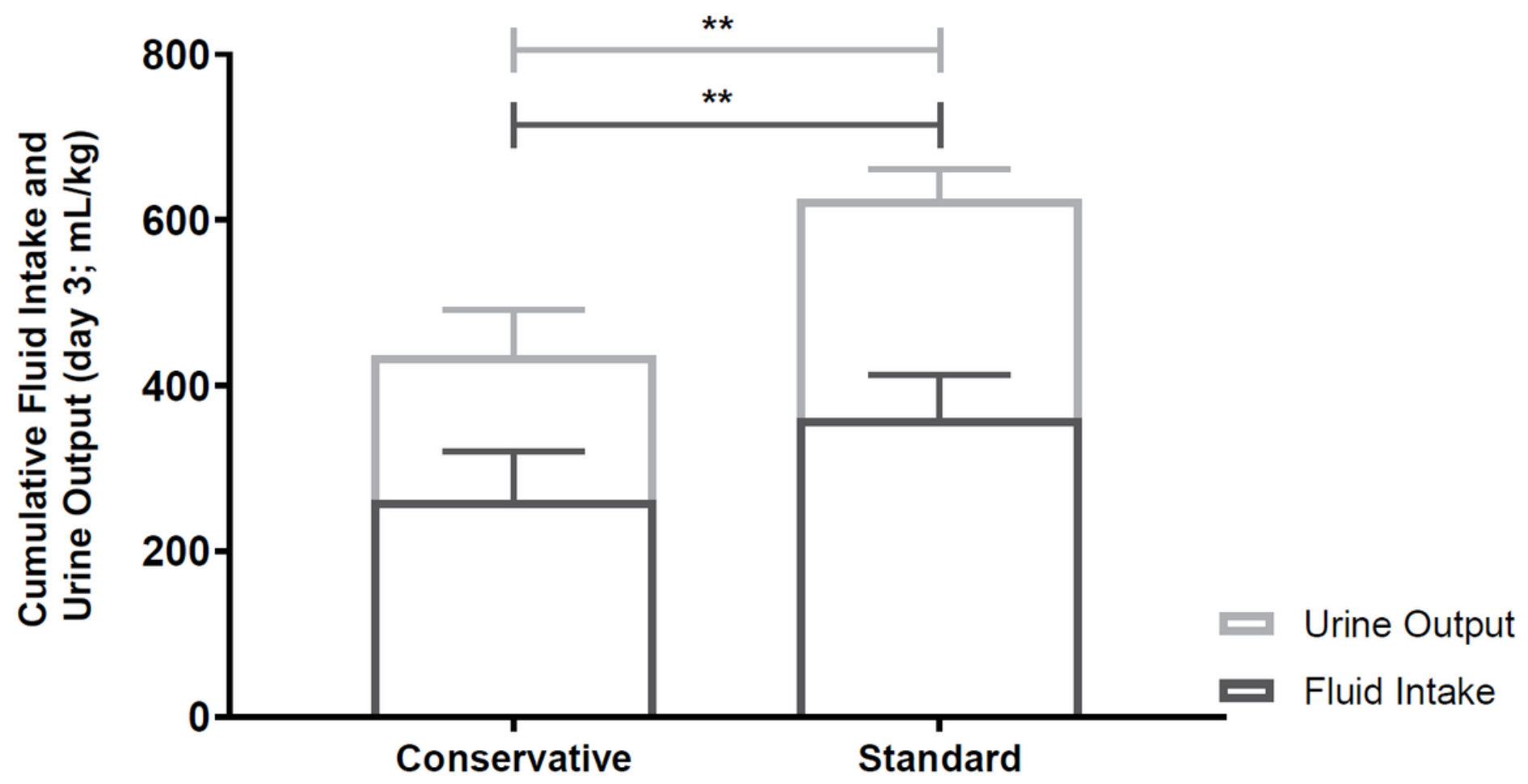

Figure 2

Cumulative Fluid intake and Urine output on Day 3 Fluid intake and urine output were significantly different between the conservative and standard fluid arm $(p<0.001)$. This did however not result in a difference in fluid balance as can be deducted from this figure. Bar graphs depict mean and SD.

\section{Supplementary Files}

This is a list of supplementary files associated with this preprint. Click to download.

- supplement1.doc 